\title{
RESPIRATORY FUNCTION AND PORTABLE OXYGEN THERAPY IN CHRONIC NON-SPECIFIC LUNG DISEASE IN RELATION TO PROGNOSIS
}

\author{
BY \\ J. E. COTES \\ From the Pneumoconiosis Research Unit of the Medical Research Council, Llandough Hospital, near Penarth, Glam.
}

(RECEIVED FOR PUBLICATION FEBRUARY 6, 1960)

Studies which relate prognosis in chronic lung disease to the results of assessment of lung function have usually been confined to a limited number of tests (Bates, Knott, and Christie, 1956; Platts and Greaves, 1957; Simpson, 1958). Such studies indicate which physiological tests have a prognostic value but provide limited information about their relative merits for prognosis. This information can only be obtained by the application to suitable subjects, both at rest and on exercise, of a number of tests which between them cover all aspects of respiratory function (cf., Baldwin, Cournand, and Richards, 1949; Gilson and Hugh-Jones, 1955). An analysis of this type is presented here. The data form part of an investigation into the ventilation changes between air and oxygen breathing on exercise in patients with chronic respiratory insufficiency. They fall into two groups: Series A, the three-and-a-half-year follow-up of 29 patients who were first assessed for their response to oxygen on exercise in 1955 (Cotes and Gilson, 1956a); Series B, the 12-month follow-up of 38 patients whose pulmonary function was studied in detail to answer questions raised by the earlier investigation. In the two series $A$ and $B, 69 \%$ and $21 \%$ of the patients respectively died during the periods of follow-up. The findings from the two investigations are consistent and suggest that for these subjects and conditions the degree of perfusion of underventilated alveoli (Riley and Cournand, 1951) is a better guide to prognosis than the pulmonary diffusing capacity, the arterial carbon dioxide tension, or any other single index of pulmonary function.

\section{SubJeCts}

The subjects were disabled coalworkers, in whom walking exercise ability was limited by breathlessness to about 200 yards on the flat (Grade 4, Fletcher, 1952). They were selected for investigation on account of this chronic respiratory insufficiency uncomplicated by lesions of other systems. Of the 29 subjects followed for three and a half years (Series A), 16 had progressive massive fibrosis of coalworkers' pneumoconiosis (Cochrane, Davies, and Fletcher, 1951), two had simple pneumoconiosis, and 11 had no radiological evidence of pneumoconiosis. All were considered clinically to have chronic bronchitis and/or emphysema and, on electrocardiography, to have some degree of right ventricular strain; a combination of right axis deviation in the standard limb leads, pulmonary $P$ waves, and a low $R$ and deep $S$ wave with upright 7 wave in $V_{6}$ was accepted as evidence for this purpose (Thomas, 1951 and 1955). Of the 38 subjects studie 9 in greater detail (Series B), 20 had progressive massive fibrosis, nine simple pneumoconiosis, and nine no evidence of pneumoconiosis; 16 showed the changes of right ventricular strain. Each assessment was carried out after a period of in-patient treatment in hospital at a time when the subject was free from clinical chest infection, reversible bronchial obstruction, congestive cardiac failure, and other conditions which might otherwise have obscured the underlying pulmonary pathophysiology.

\section{METHODS}

Each subject was studied at rest and while walking on a motor-driven treadmill which was adjusted so that he needed to stop on account of breathlessness after walking for approximately two minutes while breathing air. If he could double his walking time while breathing oxygen under these conditions he was considered to benefit from oxygen on exercise and to be a potential candidate for portable oxygen therapy; appropriate equipment (Cotes and Gilson, 1956b) was supplied on long-term loan if, in addition to being suitable, he found the equipment of assistance in the performance of everyday tasks.

In addition to assessment of exercise ability, indices of ventilatory capacity, lung volume, pulmonary diflusing capacity, and alveolar ventilation and perfusion, including arterial blood gas tensions, were determined using the following method. 
Expired gas was analysed using a low-resistance gas meter and micro Scholander gas analyser, duplicate determinations of oxygen and carbon dioxide being required to agree to $0.04 \%$. Arterial blood was sampled from an indwelling arterial cannula (Berneus, Carlsten, Holmgren, and Seldinger, 1954), and analysed for oxygen and carbon dioxide by a modified bubble method (Riley, Campbell, and Shepard, 1957). The standard deviation of a single paired determination of $\mathrm{CO}_{2}$ tension $\left(\mathrm{PaCO}_{2}\right)$ was $\pm 3.3 \mathrm{~mm}$. $\mathrm{Hg}$ and of oxygen tension $\left(\mathrm{PaO}_{2}\right) \pm 2.60 \mathrm{~mm}$. $\mathrm{Hg}$ (Cotes and Oldham, 1959). Physiological dead space, as a percentage of the tidal volume $\left(V_{D} \% V_{T}\right)$, was calculated from the arterial and mixed expired carbon dioxide tensions. Total lung capacity (T.L.C.) and its subdivisions (the functional residual capacity, F.R.C., and the residual volume, Rd.V.) were determined by a helium dilution method (Gilson and Hugh-Jones, 1949). Pulmonary diffusing capacity $\left(\mathrm{D}_{\mathrm{co}}\right)$ was measured by a modification of the single-breath method of Forster, Fowler, Bates, and Van Lingen (1954) in which the time of breath-holding was taken to include two-thirds of the inspiratory time and half the time of sample collection in order to make allowance for the changes in alveolar carbon monoxide concentration during these manceuvres (Jones and Meade, 1960). Maximum breathing capacity (M.B.C.) was determined by the indirect method of Kennedy (1953) from 40 times the 0.75 second forced expiratory volume (Gandevia and Hugh-Jones, 1957) using the apparatus described by McKerrow, McDermott, and Gilson (1960). The effectiveness of pulmonary perfusion with respect to ventilation was estimated by the method of Riley and Cournand (1951) and expressed as the percentage venous admixture $\left(Q_{\mathrm{vA}} \% \mathrm{Q}_{\mathrm{T}}\right)$. This is the proportion of mixed venous blood which, if added to blood in equilibrium with alveolar gas, within the limits imposed by the diffusing capacity, would give the observed arterial oxygen saturation; for this purpose the effective alveolar oxygen tension is calculated from the arterial carbon dioxide tension and the respiratory quotient of the expired gas. In the present application of the method, the oxygen diffusing capacity was assumed to be numerically equal to the measured single-breath diffusing capacity for carbon monoxide. This is approximately the case (Forster, Cohn, Briscoe, Blakemore, and Riley, 1955) and any discrepancy is unlikely to have materially affected the findings. The mixed venous blood oxygen saturation was not measured directly but was calculated assuming a mixed venous to pulmonary end capillary blood oxygen saturation difference of $30 \%$ for all subjects. Systematically lower or higher values for the venous admixture would have been obtained by assuming respectively a larger or smaller difference; the likelihood and possible consequences of ignoring systematic variations when making these calculations is considered below. The rate of energy expenditure on exercise was estimated from the speed and slope of the treadmill and the subject's body weight using the following relationship: energy expenditure per unit of body weight $=0.00305 \mathrm{~V}^{2}+$ $0.1884 \mathrm{I} . \mathrm{V}$. $+0.04 \mathrm{kcal}$. per $\mathrm{kg}$. per min., where I is the percentage incline of the treadmill and $V$ is the velocity in miles per hour (Cotes and Meade, 1960). This was done in preference to direct estimation from analysis of expired gas because the time of exercise was short.

Of the other indices studied, the haemoglobin percentage $(\mathrm{Hb} \%)$ was determined using the M.R.C. grey wedge photometer (King, 1947) and the erythrocyte sedimentation rate (E.S.R.) was estimated by Westergren's method.

\section{RESULTS}

SERIES A: Three-AND-A-HALF-yeAR Follow-UP of 29 PATIENTS. - The clinical and post-mortem findings on the 29 subjects who, in 1955, were studied clinically and assessed for their response to oxygen on exercise (Cotes and Gilson, 1956a) are summarized in Table I: the available physiological findings, which include the maximum breathing capacity, the maximum work rate, the haemoglobin percentage and erythrocyte sedimentation rate, are presented in Table II. These data have been analysed with respect to the presence or absence of pneumoconiosis; to whether or not subjects benefited from oxygen on exercise and whether or not they were supplied with portable oxygen apparatus.

(a) Pneumoconiosis.-For this group of disabled subjects the time of survival was not significantly different between those with and without progressive massive fibrosis (P.M.F.), though on average the time for the former was a little shorter: P.M.F. did not appear to influence the response to oxygen on exercise. The subjects have therefore all been included together, irrespective of radiological category, in considering the interpretation of these indices. The subjects with P.M.F. did differ from the remainder in that they had the higher erythrocyte sedimentation rates $(P<0.05)$ and it is likely that their blood haemoglobin levels were lower $(0.10>P>0.05)$; these differences were reflected in a negative correlation $(\mathrm{P} \bumpeq 0.05)$ between the erythrocyte sedimentation rate and the haemoglobin percentage for the group as a whole : neither index was related to prognosis or to the ability of the subjects to benefit from oxygen on exercise.

(b) Response to Oxygen on Exercise and Use of Portable Oxygen Related to Prognosis.-Twenty-one subjects were found to benefit from oxygen on exercise; during the subsequent three and a half years 18 of them died. Eight subjects failed to benefit from oxygen on exercise, but during this same period only two deaths occurred. These differences in survival are highly significant $(P<0.005)$. Table II shows that the use of portable oxygen equipment (for an average of 13 months) by eight subjects out of the 21 found to benefit from oxygen on exercise did not either lengthen or shorten 
the survival time, and that none of the other indices analysed had any significant prognostic value. Amongst those who benefited from oxygen, the subjects who were issued with oxygen sets were found subsequently to have the higher haemoglobin

TABLE I

AGE, DIAGNOSIS, RESPONSE TO OXYGEN ON EXERCISE, SURVIVAL TIME, AND CAUSE OF DEATH OF SUBJECTS IN SERIES A

\begin{tabular}{|c|c|c|c|c|c|}
\hline $\begin{array}{l}\text { Case } \\
\text { No. }\end{array}$ & $\left|\begin{array}{c}\text { Age } \\
(\mathrm{yr} .)\end{array}\right|$ & $\begin{array}{c}\text { Sur- } \\
\text { vival } \\
(\mathrm{mth})\end{array}$ & Diagnosis & $\begin{array}{c}\text { Res- } \\
\text { ponse to } \\
\mathrm{O}_{2} \text { on } \\
\text { Exercise }\end{array}$ & $\begin{array}{c}\text { Immediate Cause } \\
\text { of Death }\end{array}$ \\
\hline A. 1 & 69 & 10 & CB: E. RH & Yes & $\begin{array}{l}\text { Respiratory and } \\
\text { congestive cardiac } \\
\text { failure }\end{array}$ \\
\hline 2 & 65 & $>42$ & CB: E. & No & - \\
\hline 3 & 55 & $>42$ & $\begin{array}{l}\text { CB: Pnc. (-/B.4/4) } \\
\text { RH }\end{array}$ & ", & - \\
\hline $\begin{array}{l}4 \\
5 \\
6\end{array}$ & $\begin{array}{l}64 \\
49 \\
64\end{array}$ & $\begin{array}{r}26 \\
6 \\
32\end{array}$ & $\begin{array}{l}\text { CB: Pnc. }(2 / \mathrm{A} .3 / 1) \\
\text { CB: Pnc. }(-\mathrm{D} .4 / 5) \\
\text { CB: Pnc. }(-\mathrm{D} .3 / 2)\end{array}$ & $\begin{array}{l}\text { Yes } \\
\text { No }\end{array}$ & $\begin{array}{l}\text { Not known } \\
\text { Suicide } \\
\text { Bronchopneumonia }\end{array}$ \\
\hline 7 & 58 & 2 & $\begin{array}{c}\text { CB:E.Pnc. } \\
(-/ \text { C.4/4) }\end{array}$ & Yes & $\begin{array}{l}\text { Respiratory failure } \\
\text { associated with } \\
\text { purulent bron- } \\
\text { chitis }\end{array}$ \\
\hline 8 & 60 & 32 & $\begin{array}{l}\text { CB: E. Pnc. } \\
(2 / A .2 /-)\end{array}$ & Yes. P. & $\begin{array}{l}\text { Respiratory and } \\
\text { congestive cardiac } \\
\text { failure }\end{array}$ \\
\hline 9 & 62 & 7 & $\begin{array}{l}\text { CB: Pnc. }(-D .5 / 5) \\
\text { RH }\end{array}$ & Yes & $\begin{array}{l}\text { Pulmonary artery } \\
\text { thrombosis }\end{array}$ \\
\hline 10 & 36 & 3 & E. & ," & $\begin{array}{l}\text { Respiratory failure } \\
\text { associated with } \\
\text { purulent bron- } \\
\text { chitis }\end{array}$ \\
\hline 11 & $\begin{array}{l}55 \\
62\end{array}$ & $>42$ & CB. RH & No $p$ & - \\
\hline 12 & 63 & 10 & $\begin{array}{l}\text { CB: Pnc. }(-D .4 / 3) \\
\text { RH }\end{array}$ & Yes. P. & $\begin{array}{l}\text { Pulmonary artery } \\
\text { thrombosis }\end{array}$ \\
\hline 13 & 56 & 26 & $\begin{array}{l}\text { CB: Pnc. (3/B.3/0) } \\
\text { RH }\end{array}$ & , , , & $\begin{array}{l}\text { Respiratory failure } \\
\text { associated with } \\
\text { purulent bron- } \\
\text { chitiscomplicating } \\
\text { Asian 'flu }\end{array}$ \\
\hline $\begin{array}{l}14 \\
15\end{array}$ & $\begin{array}{l}66 \\
60\end{array}$ & $\begin{array}{l}30 \\
19\end{array}$ & $\begin{array}{l}\text { CB: E. } \\
\text { CB: E.Pnc. }\end{array}$ & Yes & Not known \\
\hline 16 & 61 & 32 & $\begin{array}{l}(-/ \mathrm{C} .4 / 4) \\
\text { CB: E. }\end{array}$ & , & Congestive cardiac \\
\hline 17 & 70 & $>42$ & CB: & No & failure \\
\hline 18 & 53 & $>42$ & $\begin{array}{l}\text { CB: } \\
\text { RH }\end{array}$ & ", & $\overline{-}$ \\
\hline $\begin{array}{l}19 \\
20\end{array}$ & $\begin{array}{l}64 \\
71\end{array}$ & $\begin{array}{l}>42 \\
>42\end{array}$ & $\begin{array}{l}\text { CB: } \\
\text { CB: Pnc. }(-/ D .4 / 6) \\
\text { RH }\end{array}$ & Ÿes & 二 \\
\hline 21 & 50 & 5 & $\begin{array}{l}\text { CB:Pnc. }(-/ C .5 / 4) \\
\text { RH }\end{array}$ & Yes. P. & $\begin{array}{l}\text { Respiratory failure } \\
\text { associated with } \\
\text { purulent bron- } \\
\text { chitis }\end{array}$ \\
\hline 22 & 64 & 16 & $\begin{array}{l}\text { CB: Pnc. (3/B.5/2) } \\
\text { RH }\end{array}$ & " " & $\begin{array}{l}\text { Respiratory failure } \\
\text { associated with } \\
\text { purulent bron- } \\
\text { chitis }\end{array}$ \\
\hline 23 & 63 & 34 & CB: Pnc. (-/D.3/2) & No & $\begin{array}{l}\text { Respiratory and } \\
\text { congestive cardiac } \\
\text { failure }\end{array}$ \\
\hline 24 & 35 & $>42$ & CB: Pulm.fib.: & Yes & - \\
\hline 25 & 54 & 28 & CB: & Yes. P. & $\begin{array}{l}\text { Pulmonary embol- } \\
\text { ism from femoral } \\
\text { veins }\end{array}$ \\
\hline 26 & 66 & 8 & 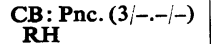 & $"$, & Respiratory failure \\
\hline $\begin{array}{l}27 \\
28\end{array}$ & $\begin{array}{l}66 \\
62\end{array}$ & $\begin{array}{r}>42 \\
31\end{array}$ & $\begin{array}{l}\text { CB:Pnc. }(2 /--\mid-1-) \\
\text { CB: E. RH }\end{array}$ & Yës " & $\begin{array}{l}\text { Bronchopneumonia } \\
\text { and cerebral }\end{array}$ \\
\hline 29 & 53 & 22 & CB: Pnc. (3/D.5/3) & ", & Not known \\
\hline
\end{tabular}

$\mathrm{CB}=$ chronic bronchitis. $\mathrm{E}=$ advanced emphysema. Pnc. $=$ Pneumoconiosis (radiological category in parentheses). Pulm. fib.= Pneumoconiosis (radiological category in parentheses). Pulm. fib. $=$
Pulmonary fibrosis. $\mathbf{A}=$ Infective asthma. $\mathbf{P}=$ Supplied with portable Pulmonary fibrosis. $A=$ Infective asthma. $P=$ Supplied with portable
oxygen equipment. disease.
TABLE II

MEAN VALUES FOR INDICES ANALYSED WITH RESPECT TO PROGRESSIVE MASSIVE FIBROSIS, RESPONSE TO OXYGEN ON EXERCISE, AND SUITABILITY FOR PORTABLE OXYGEN APPARATUS IN SERIES A

\begin{tabular}{|c|c|c|c|c|c|c|c|}
\hline & \multicolumn{3}{|c|}{$\begin{array}{l}\text { Radiological } \\
\text { Category }\end{array}$} & \multicolumn{4}{|c|}{$\begin{array}{c}\text { Response to Oxygen on } \\
\text { Exercise }\end{array}$} \\
\hline & \multirow[b]{2}{*}{ 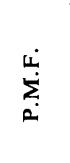 } & \multirow[b]{2}{*}{ 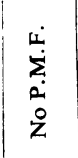 } & \multirow[b]{2}{*}{ Total } & \multirow[b]{2}{*}{$\begin{array}{c}\text { No } \\
\text { Bene- } \\
\text { fit }\end{array}$} & \multicolumn{2}{|c|}{ Benefit } & \multirow[b]{2}{*}{ Total } \\
\hline & & & & & $\begin{array}{c}\text { No } \\
\text { Port- } \\
\text { able } \\
\mathrm{O}_{2}\end{array}$ & $\left|\begin{array}{c}\text { Port- } \\
\text { able } \\
\mathrm{O}_{2} \text { Set } \\
\text { Pro- } \\
\text { vided }\end{array}\right|$ & \\
\hline $\begin{array}{l}\text { Number } \\
\text { Age (yr.) } \\
\text { Weight (kg.) } \\
\text { E.S. R. (mm. } \\
\text { Hb ( } \% \text {. } \\
\text { Survival number } \\
\text { Time in months }\end{array}$ & $\begin{array}{l}16 \\
59 \cdot 1 \\
53 \cdot 5 \\
29 \cdot 6^{*} \\
97 \cdot 1 \\
3 \\
22 \cdot 7 \\
(\max . \\
\text { mon }\end{array}$ & \begin{tabular}{|c|}
13 \\
$59 \cdot 2$ \\
$55 \cdot 5$ \\
$14 \cdot 5 *$ \\
$105 \cdot 2$ \\
6 \\
$30 \cdot 3$ \\
$=42$ \\
ths)
\end{tabular} & $\begin{array}{c}29 \\
59 \cdot 1 \\
54.4 \\
22 \cdot 8 \\
100 \cdot 7 \\
9 \\
26 \cdot 1\end{array}$ & $\begin{array}{c}8 \\
61 \cdot 1 \\
54 \cdot 3 \\
23 \cdot 5 \\
97 \cdot 0 \\
6 \\
39 \cdot 8 *\end{array}$ & \begin{tabular}{|l|}
3 \\
$57 \cdot 4$ \\
$52 \cdot 7$ \\
$28 \cdot 0$ \\
$96 \cdot . * *$ \\
2 \\
20.9
\end{tabular} & \begin{tabular}{|c|}
8 \\
$59 \cdot 9$ \\
$57 \cdot 3$ \\
$13 \cdot 6$ \\
$110 \cdot 6 *$ \\
1 \\
$20 \cdot 9$
\end{tabular} & $\begin{array}{l}21 \\
58 \cdot 3 \\
54 \cdot 4 \\
22 \cdot 5 \\
102 \\
3 \\
20 \cdot 9 *\end{array}$ \\
\hline $\begin{array}{l}\text { Maximum exer- } \\
\text { cise ability } \\
\text { (kcal./min.) } \\
\text { Indirect M.B.C. } \dagger \\
\text { (1./min.) } \\
\text { number }\end{array}$ & $\begin{array}{c}3 \cdot 09 \\
22 \cdot 2 \\
(12)\end{array}$ & $\begin{array}{c}3 \cdot 01 \\
19 \cdot 1 \\
(9)\end{array}$ & $\begin{array}{l}3 \cdot 05 \\
20 \cdot 9 \\
(21)\end{array}$ & $\begin{array}{c}2.57 \\
18 \cdot 8 \\
(8)\end{array}$ & $\begin{array}{c}2 \cdot 95 \\
24 \cdot 5 \\
(6)\end{array}$ & $\begin{array}{c}3 \cdot 71 \\
19 \cdot 0 \\
(7)\end{array}$ & $\begin{array}{l}3 \cdot 24 \\
21 \cdot 6 \\
(13)\end{array}$ \\
\hline
\end{tabular}

${ }^{*} \mathbf{P}<0.05$. + This measurement was not made on all subjects.

percentages $(\mathrm{P} \bumpeq 0.01)$ and lower erythrocyte sedi- $\vec{\oplus}$ mentation rates $(0.10>\mathrm{P}>0.05)$. Considering the survivors, of the three subjects who responded oxygen in 1955 and are still alive, two (Nos. 20 an 27) stand out because of the excellence of the atte tion in their homes; the third (No. 24) is a much younger man. Of the six survivors who failed to $\stackrel{\frac{\Omega}{\perp}}{\propto}$ benefit from oxygen in 1955 , four have been re- $\vec{F}$ assessed (Nos. 11, 17, 18, and 19) and, of these, two 응 (Nos. 17 and 18) now respond to oxygen on exercise, $כ$ in both cases following a long period of chest illness. One subject (No. 2) has since died of respiratory and congestive cardiac failure after repeated chest $\overline{0}$ illnesses, and one (No. 3), who has had a laryngec- $\underset{x}{x}$ tomy for malignant disease, has not been reassessed. $\ddot{-}$ In summary, by the time a man benefits from oxygen on exercise he tends to have a poor prognosis. 8

SERIES B : ONe-YeAR Follow-UP of 38 PATIEnts.

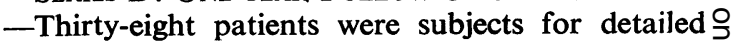
clinical and physiological assessment in the period $>$ March-August, 1958. The findings are summarized in Tables III-VII and are considered below.

(i) Radiological Category.-The findings analysed with respect to the radiographic category of pneumoconiosis are presented in Table 1II. As in $\omega$ the earlier group, there is a significant negative correlation between the haemoglobin percentage and the erythrocyte sedimentation rate, the subjects $\Phi_{\infty}$ with progressive massive fibrosis having the highest ${ }^{+}$ E.S.R.s. They also have the smallest total lung capacities and residual volumes. In the case of all $\stackrel{\mathrm{D}}{\mathrm{D}}$ 
TABLE III

INDICES OF RESPIRATORY FUNCTION AT REST IN 38 SUBJECTS ANALYSED BY RADIOLOGICAL CATEGORY OF PNEUMOCONIOSIS (MEAN VALUES)

\begin{tabular}{|c|c|c|c|c|c|c|}
\hline & & & P.M.F. & $\begin{array}{l}\text { Simple } \\
\text { Pneumo- } \\
\text { coniosis }\end{array}$ & $\begin{array}{l}\text { Without } \\
\text { Pneumo- } \\
\text { coniosis }\end{array}$ & All \\
\hline $\begin{array}{ll}\text { Number } & \ldots \\
\text { Age (years) } & \ldots \\
\text { Weight (kg.) } & \ldots\end{array}$ & $\begin{array}{l}\cdots \\
\cdots \\
\cdots\end{array}$ & $\begin{array}{l}\cdots \\
\cdots \\
\cdots\end{array}$ & $\begin{array}{l}20 \\
55 \cdot 0 \\
60 \cdot 2\end{array}$ & $\begin{array}{l}9 \\
60 \cdot 4 \\
56 \cdot 1\end{array}$ & $\begin{array}{l}9 \\
50 \cdot 9 \\
61 \cdot 1\end{array}$ & $\begin{array}{l}38 \\
55 \cdot 3 \\
59 \cdot 4\end{array}$ \\
\hline \multicolumn{3}{|c|}{$\begin{array}{l}\text { Indirect M B.C. (1. min.) } \ldots \\
\% \text { increase in M.B.C. af.er } \\
\text { adrenaline .. }\end{array}$} & $\begin{array}{l}28 \cdot 5 \\
22 \cdot 4\end{array}$ & $\begin{array}{l}26 \cdot 6 \\
26 \cdot 6\end{array}$ & $\begin{array}{l}29 \cdot 8 \\
19 \cdot 7\end{array}$ & $\begin{array}{l}28 \cdot 3 \\
22 \cdot 6\end{array}$ \\
\hline $\begin{array}{l}\text { VE (air) }(1 . / \mathrm{min} .) \\
\mathrm{PaCO}_{2}(\mathrm{~mm} . \mathrm{Hg}) \\
\mathrm{VD}_{0} \% \mathrm{VT} \\
\mathrm{PaO}_{2}(\mathrm{~mm} . \mathrm{Hg})\end{array}$ & $\begin{array}{l}\cdots \\
\cdots \\
\cdots \\
\cdots\end{array}$ & $\begin{array}{l}\cdots \\
\cdots \\
\cdots \\
\cdots\end{array}$ & $\begin{array}{l}10 \cdot 8 \\
41 \cdot 5 \\
44 \cdot 0 \\
65 \cdot 5\end{array}$ & $\begin{array}{l}10 \cdot 2 \\
43 \cdot 7 \\
50 \cdot 7 \\
64 \cdot 8\end{array}$ & $\begin{array}{r}9 \cdot 8 \\
41 \cdot 7 \\
44 \cdot 1 \\
68 \cdot 3\end{array}$ & $\begin{array}{l}10 \cdot 4 \\
42 \cdot 0 \\
45 \cdot 6 \\
66 \cdot 0\end{array}$ \\
\hline $\begin{array}{l}\text { Qva \% QT } \\
\text { Dco (ml. min. } \mathrm{mm} \text {. }\end{array}$ & $\dot{H g})$ & $\begin{array}{l}\cdots \\
\cdots\end{array}$ & $\begin{array}{l}20 \cdot 7 \\
14 \cdot 9\end{array}$ & $\begin{array}{l}19 \cdot 9 \\
13 \cdot 1\end{array}$ & $\begin{array}{l}19 \cdot 4 \\
19 \cdot 6\end{array}$ & $\begin{array}{l}20 \cdot 1 \\
15 \cdot 5\end{array}$ \\
\hline $\begin{array}{l}\text { T.L.C. }(1 .)^{*} \\
\text { F.R.C. }(1 .)^{*} \\
\text { Rd. V. }(1 .)^{*} \\
\text { Rd. V \% T.L.C. }\end{array}$ & $\begin{array}{l}\cdots \\
\cdots \\
\cdots\end{array}$ & $\begin{array}{l}\cdots \\
\cdots \\
\cdots\end{array}$ & $\begin{array}{r}6 \cdot 20 \\
4 \cdot 34 \\
3 \cdot 38 \\
54 \cdot 1\end{array}$ & $\begin{array}{r}6 \cdot 89 \\
5 \cdot 15 \\
4 \cdot 17 \\
60 \cdot 1\end{array}$ & $\begin{array}{r}8 \cdot 01 \\
5 \cdot 95 \\
4 \cdot 71 \\
58 \cdot 3\end{array}$ & $\begin{array}{r}6 \cdot 76 \\
4 \cdot 88 \\
3 \cdot 85 \\
56 \cdot 4\end{array}$ \\
\hline $\begin{array}{l}\text { Hb }(\%)^{*} \\
\text { E.S.R. }(\mathrm{mm} . \dot{\mathrm{hr}})^{*}\end{array}$ & $\begin{array}{l}\cdots \\
\cdots\end{array}$ & $\begin{array}{l}\cdots \\
\cdots\end{array}$ & $\begin{array}{l}97 \cdot 1 \\
33 \cdot 4\end{array}$ & $\begin{array}{r}101 \cdot 3 \\
20 \cdot 6\end{array}$ & $\begin{array}{r}107 \cdot 1 \\
11 \cdot 2\end{array}$ & $\begin{array}{r}100 \cdot 4 \\
25 \cdot 1\end{array}$ \\
\hline
\end{tabular}

*=Significant differences between subgroups.

other indices, the subjects in the different radiological categories do not differ significantly from each other and they have therefore been considered together in the following analysis.

(ii) Pulmonary Heart Disease.-When analysed with respect to pulmonary heart disease, the subjects with some degree of right ventricular strain differ in a number of respects $(\mathrm{P}<0.05)$ from those with more normal electrocardiographs (Table JV). Those with heart disease are lighter in

TABLE IV

AVERAGE VALUES FOR INDICES OF PULMONARY FUNCTION WHICH DIFFER $(\mathrm{P}<0.05)$ BETWEEN SUBJECTS WITH AND WITHOUT PULMONARY HEART DISEASE

\begin{tabular}{|c|c|c|c|}
\hline \multirow{2}{*}{\multicolumn{2}{|c|}{ Index }} & \multicolumn{2}{|c|}{$\begin{array}{c}\text { Pulmonary } \\
\text { Heart Disease }\end{array}$} \\
\hline & & Present & Absent \\
\hline 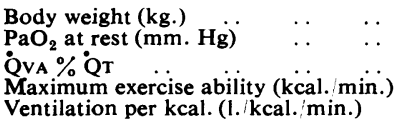 & \begin{tabular}{l|l}
$\cdots$ \\
$\cdots$ \\
$\cdots$ \\
$\cdots$
\end{tabular} & $\begin{array}{c}54 \cdot 2 \\
62 \cdot 0 \\
23 \cdot 9 \\
2 \cdot 82 \\
9 \cdot 48\end{array}$ & $\begin{array}{c}63 \cdot 3 \\
68 \cdot 7 \\
17 \cdot 8 \\
4 \cdot 14 \\
6 \cdot 99\end{array}$ \\
\hline
\end{tabular}

weight and at rest their arterial oxygen tensions are lower and calculated venous admixtures higher; on exercise, maximum exercise ability is decreased, and exercise ventilation per unit of energy expenditure is increased.

(iii) Response to Oxygen on Exercise.--Nineteen of the 38 subjects were found at the time of assessment to benefit from oxygen on exercise. At rest these subjects differ from those who failed to benefit only in that they have a higher venous admixture (Table V). On exercise, breathing air, the arterial blood oxygen tension drops in these subjects, and on exercise, breathing oxygen, the arterial blood carbon dioxide tension rises. Other changes also take place which are considered elsewhere (Cotes, 1960).

\section{TABLE V}

AVERAGE VALUES FOR INDICES OF PULMONARY FUNCTION WHICH DIFFER $(P<0.05)$ BETWEEN SUBJECTS RESPONDING AND NOT RESPONDING TO OXYGEN ON EXERCISE

\begin{tabular}{|c|c|c|}
\hline & \multicolumn{2}{|c|}{$\begin{array}{l}\text { Response to Oxygen } \\
\text { on Exercise }\end{array}$} \\
\hline & Benefit & No Benefit \\
\hline 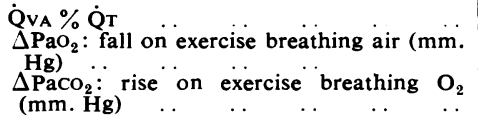 & $\begin{array}{r}10 \cdot 2 \\
7 \cdot 1\end{array}$ & $\begin{array}{r}17 \cdot 2 \\
2 \cdot 7 \\
2 \cdot 4\end{array}$ \\
\hline
\end{tabular}

Findings in Relation to Prognosis.-Sixteen subjects out of a total of 67 in the two series died within 12 months of assessment; their clinical features are to be found in Tables I and VI for Series A and B respectively. The gross post-mortem findings are also recorded. Preliminary analysis of large lung sections (Gough and Wentworth, 1949) shows examples of emphysema of the diffuse, centrilobular (Leopold and Gough, 1957), and destructive types, but these subdivisions do not appear to be distinguishable by the physiological techniques applied. The one-year mortality experience in these cases is analysed in Table VII with respect to the presence or absence of pulmonary

TABLE VI

SUMMARY OF FINDINGS IN 8 PATIENTS DYING WITHIN 12 MONTHS OF ASSESSMENT

\begin{tabular}{|c|c|c|c|c|c|}
\hline No. & $\begin{array}{c}\text { Age } \\
\text { (yr.) }\end{array}$ & $\begin{array}{c}\text { Diagnosis } \\
\text { on Assessment }\end{array}$ & $\begin{array}{l}\text { Res- } \\
\text { ponse } \\
\text { to } \mathrm{O}_{2}\end{array}$ & $\begin{array}{c}\text { Sur- } \\
\text { vival } \\
\text { (mth.) }\end{array}$ & $\begin{array}{c}\text { Immediate } \\
\text { Cause of Death }\end{array}$ \\
\hline B. 5 & $\begin{array}{l}47 \\
60\end{array}$ & $\begin{array}{l}\text { E. Rt. Ht. } \\
\text { Pnc. }(- \text { C.6). Rh. }\end{array}$ & $\begin{array}{c}\text { Yes } \\
.,\end{array}$ & $\begin{array}{r}12 \\
8\end{array}$ & Respiratory failure \\
\hline 18 & 56 & $\begin{array}{l}\text { Pnc. }(- \text { C.4 5). E. } \\
\text { Rt. Ht. }\end{array}$ & No & 7 & $\begin{array}{l}\text { Congestive heart } \\
\text { failure }\end{array}$ \\
\hline 19 & 44 & $\begin{array}{l}\text { Pnc. }(- \text { C.5 4). Rt. } \\
\text { Ht.f. }\end{array}$ & Yes & $3 \frac{1}{2}$ & $\begin{array}{l}\text { Respiratory and } \\
\text { congestive cardiac } \\
\text { failure }\end{array}$ \\
\hline 22 & 46 & $\begin{array}{l}\text { Pnc. (-D.5 4). Rt. } \\
\text { Ht. }\end{array}$ & " & 3 & Respiratory failure \\
\hline 32 & 54 & $\begin{array}{l}\text { Pnc. }(2-1-) \text {. E., } \\
\text { Rt. Ht.f. }\end{array}$ & 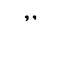 & $3 \frac{1}{2}$ & $\begin{array}{l}\text { Respiratory failure } \\
\text { associated with } \\
\text { purulent bronchitis }\end{array}$ \\
\hline 34 & 42 & $\begin{array}{l}\text { Pnc. }(-C .42) . \text { Rt. } \\
\text { Ht. }\end{array}$ & ", & 5 & $\begin{array}{l}\text { Respiratory and } \\
\text { congestive cardiac } \\
\text { failure }\end{array}$ \\
\hline 35 & 59 & $\begin{array}{l}\text { Pnc. }(2 / C .4 / 2) . \text { Rt. } \\
\text { Ht. }\end{array}$ & , & 4 & $\begin{array}{l}\text { Respiratory failure } \\
\text { associated with } \\
\text { purulent bronchitis }\end{array}$ \\
\hline
\end{tabular}

$\mathrm{E}=$ advanced emphysema. Rt. Ht.=E.C.G. evidence of pulmonary heart disease. Pnc. = Category of pneumoconiosis. Rh. A. = Rheumatoid arthritis. Rt. Ht.f.=Congestive cardiac failure. 
TABLE VII

TWELVE MONTH MORTALITY EXPERIENCE IN 67 SUBJECTS WITH CHRONIC LUNG DISEASE

\begin{tabular}{|c|c|c|}
\hline \multirow{3}{*}{ Factor } & \multirow{2}{*}{\multicolumn{2}{|c|}{$\begin{array}{c}\text { Percentage Mortality } \\
\text { Factor }\end{array}$}} \\
\hline & & \\
\hline & Present & Absent \\
\hline \multirow{4}{*}{$\begin{array}{l}\text { 1. E.C.G. diagnosed pulmonary heart disease } \\
\text { 2. Improvement in exercise performance on } \\
\text { oxygen breathing } \\
\text { 3. Presence or absence of both pulmonary } \\
\text { heart disease and response to oxygen } \\
\text { 4. Progressive massive fibrosis of pneumo- } \\
\text { coniosis ... ... . . . }\end{array}$} & $26 \cdot 7^{*}$ & $5 \cdot 3$ \\
\hline & $33.8 *$ & $2 \cdot 3$ \\
\hline & $49 \cdot 5^{*}$ & 0 \\
\hline & $18 \cdot 8^{*}$ & $9 \cdot 8$ \\
\hline
\end{tabular}

heart disease, progressive massive fibrosis, and response to oxygen on exercise. In the subjects who died within 12 months of assessment, pulmonary heart disease was diagnosed more commonly and exercise performance was improved on oxygen breathing more frequently than would have been anticipated by chance $(P<0.001)$. The presence of the two criteria together was associated with a $50 \%$ mortality within 12 months in the subjects studied: no subject died amongst those in whom neither factor was present. The numbers so far available are too small to decide which of the two criteria in isolation is of greater consequence. The presence of progressive massive fibrosis did not significantly affect mortality, though it is of interest that all the deaths in the subjects with P.M.F. occurred amongst those with radiologically advanced disease (Categories $\mathrm{C}$ and $\mathrm{D}$ ).

Indices of pulmonary function, which were obtained in all subjects and in subjects in Series B alone, are analysed with respect to prognosis in Tables VIII and IX respectively. The subjects who

TABLE VIII

MEAN VALUES FOR INDICES OBTAINED IN 16 SUBJECTS DYING OF CHRONIC LUNG DISEASE WITHIN 12 MONTHS OF ASSESSMENT COMPARED WITH 51 SURVIVORS

\begin{tabular}{|c|c|c|c|c|c|c|}
\hline \multirow{2}{*}{\multicolumn{4}{|c|}{ Index }} & \multicolumn{2}{|c|}{ Mean Values } & \multirow{2}{*}{$\begin{array}{l}\text { Probability } \\
(\text { if }<0 \cdot 10)\end{array}$} \\
\hline & & & & \multirow[b]{2}{*}{$\begin{array}{c}\text { Dead } \\
16 \\
53 \cdot 8 \\
49 \cdot 5 \\
102.9 \\
25 \cdot 3 \\
22.7 \\
2 \cdot 50\end{array}$} & \multirow[b]{2}{*}{$\begin{array}{c}\text { Living } \\
51 \\
57.9 \\
59.7 \\
99.8 \\
23.7 \\
27.8 \\
3.62\end{array}$} & \\
\hline $\begin{array}{l}\text { Number } \\
\text { Age (years) } \\
\text { Weight (kg.) } \\
\text { Hb (\%) } \\
\text { E.S.R. (Weste } \\
\text { M.B.C. indire } \\
\text { Maximum wo }\end{array}$ & $\begin{array}{l}\because \\
\therefore \\
\therefore \\
\text { ergren m } \\
\text { ect } \\
\text { rk rate } \\
\text { (1 }\end{array}$ & $\begin{array}{c}\ldots \\
\cdots \\
\cdots \\
\text { hour) } \\
1 . \text { min.) }\end{array}$ & $\begin{array}{l}. \\
\ldots \\
\ldots \\
\ldots \\
\ldots\end{array}$ & & & $\begin{array}{l}\overline{\overline{1}} \\
0.001 \\
\overline{-} \\
\overline{\overline{0}} \\
0.0002\end{array}$ \\
\hline
\end{tabular}

died were of comparable age to those who survived but were significantly lighter in weight. Maximum exercise ability was less, and the levels of arterial blood oxygen were lower both at rest and on exercise. The venous admixture was greatly increased, suggesting increased perfusion of poorly ventilated alveoli. Differences between the two groups of subjects which were suggestive but which did not reach the $5 \%$ level of significance were 0 observed for the ventilation per unit of energy expenditure on exercise, the pulmonary diffusing $\frac{\bar{s}}{}$ capacity and the arterial blood carbon dioxide $\stackrel{\mathbb{Q}}{\Omega}$ tension on exercise breathing oxygen. Similar values between the two groups of subjects we $e$ s obtained for the arterial blood carbon dioxide $\vec{\circ}$ tensions with the subjects breathing air, both at $\overrightarrow{\vec{\omega}}$ rest and on exercise, for the maximum breathing $\omega^{\circ}$ capacity, the total lung volume and its subdivisions, the E.S.R. and the haemoglobin percentage.

TABLE IX

MEAN VALUES FOR INDICES OF LUNG VOLUME AND A RESPIRATORY FUNCTION IN 8 SUBJECTS DYING WITHIN 12 MONTHS OF ASSESSMENT COMPARED WITH $30 \mathrm{O}$

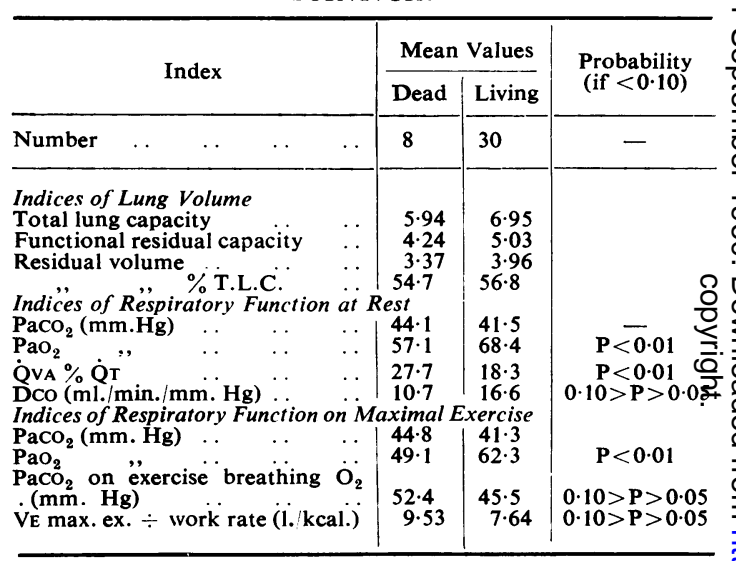

\section{Discussion}

In the present study subjects were investigated after a period of in-patient therapy at a time when their pulmonary function was likely to have been at its best; the findings may therefore differ from those of investigations carried out either haphazardly in relation to out-patient attendances or during acute illnesses.

This study includes patients with progressive $\frac{D}{O}$ massive fibrosis of coalworkers' pneumoconiosis, who had a smaller total lung capacity, a smaller $\tilde{o}^{\circ}$ residual volume, a lower haemoglobin, and a higher blood sedimentation rate than men with simple pneumoconiosis or with no pneumoconiosis. In the present series, neither the lung volume nor the blood changes relate to prognosis (cf., West, Baldwin, Cournand, and Richards, 1951; Sinclair, 1955). As the residual volume as a percentage of the total lung capacity did not differ between those with and without massive fibrosis, it seems probable that 
the fibrosis acts largely because it occupies space. The raised sedimentation rate is probably the result of the infective nature of P.M.F. (Cochrane, Miall, Clarke, Jarman, Jonathan, and Moore, 1956) and is not an indication of a more severe bronchial infection. Thus it seems probable that the remaining parts of the lung in the men with progressive massive fibrosis are damaged in much the same way as in subjects with chronic nonspecific chest disease (Fletcher, 1959). The findings for both groups of subjects may therefore reasonably be combined.

Three indices of prognostic importance emerge from this study. First, hypoxaemia and a greatly increased venous admixture; second, the ability to perform more exercise breathing oxygen than breathing air under standard conditions; and, third, E.C.G. changes characteristic of pulmonary heart disease. Each factor may occur in isolation, but more often there is a causal relationship between them. On the one hand, hypoxaemia increases pulmonary vascular resistance and hence the work required of the right heart (Cournand, 1957); on the other, correction of hypoxaemia by oxygen administration might reasonably be expected to increase exercise ability, though the manner in which it does so is not fully understood (Cotes, 1960).

The arterial oxygen tension fell on exercise by the same amount in the subjects who died as in those who survived the 12-month period of follow.up. Thus, over the short term, only the level of oxygen tension was of prognostic significance. This finding does not support the belief that a fall in arterial blood oxygen on exercise is a grave prognostic sign (Arnott, 1960). However, because those who died in the present study had lower absolute values, the changes on exercise took place on a more linear part of the oxygen dissociation curve; thus when the fall is expressed as saturation instead of tension it is a little greater than in the subjects who survived. Over a longer period, a reduction in arterial oxygen tension on exercise was itself of prognostic value as it occurred in those whose exercise performance improved on oxygen breathing; 18 of 21 such subjects died within three and a half years.

The present analysis suggests that hypoxaemia is due to perfusion of underventilated alveoli since the calculated venous admixture is increased. Because of the method of calculation, a similar result could have been obtained from a reduction in cardiac output, but this seems unlikely since the subjects were capable of increasing their cardiac output above the resting level in response to exercise.
A high venous admixture is evidence of failure of the normal mechanisms of compensation whereby pulmonary blood flow is diverted from poorlyventilated to well-ventilated regions of the lung. A minimal increase is detectable in healthy young subjects as a result of changes in posture (Riley, Permutt, Said, Godfrey, Cheng, Howell, and Shepard, 1959); age has an adverse effect (Tenney and Miller, 1956) and changes can be demonstrated following procedures which affect the pulmonary circulation in a variety of conditions (Halmagyi and Cotes, 1959). The present study suggests that the degree of inequality of perfusion with respect to ventilation is of major prognostic significance. It is likely that this can increase independent of ventilatory ability and of exercise performance breathing air, so that a subject's prognosis may deteriorate without there necessarily being any change in his symptoms or grade of breathlessness. A number of factors are likely to contribute to the development of this condition; these are reflected in the varying findings at necropsy. However, the final common pattern of function suggests that there is an underlying similarity, probably a reduction in area of gas exchange.

The factors contributing to ventilation perfusion inequality may affect indices such as the arterial carbon dioxide tension and the pulmonary diffusing capacity, which also have prognostic significance. Thus Platts and Greaves (1957) found that a rise in arterial carbon dioxide during a chest illness was of some prognostic importance, but this index is of limited usefulness since it may return to normal on recovery. The present findings suggest that a latent tendency to hypoventilation persists and that it can be made more obvious by exercising the subject while he is breathing oxygen.

The findings for the carbon monoxide diffusing capacity by the single breath method are in line with the observations of Bates et al. (1956), who used the steady-state method and obtained significantly lower values in subjects with emphysema than in those with chronic bronchitis without dyspnoea on exertion. But in the patients with emphysema they were not able to separate those with a poor prognosis by this means. The singlebreath method may provide a better index and its usefulness is likely to be increased when measurements of the diffusion characteristics of the alveolar capillary membrane are separated from those of the volume of blood in the alveolar capillaries (Roughton and Forster, 1957). There is little information about the long-term behaviour of these indices in patients with chronic non-specific lung disease, and I hope that those who have already been investigated 
will be reassessed in the future (e.g., Ogilvie, 1959; Shepard, Cohn, Cohen, A.rmstrong, Carroll, Donoso, and Riley, 1955).

Improvement in exercise performance on oxygen breathing led us to issue portable oxygen equipment in selected cases. Its use does not appear to have affected prognosis, but the subjects issued with oxygen equipment were not strictly comparable with those who benefited from oxygen to a lesser extent. Thus the former had higher haemoglobin percentages and lower erythrocyte sedimentation rates and, in Series B, they also had higher venous admixtures $(P<0.05)$. Whether such subjects will live longer without access to extra oxygen on exercise can only be determined accurately by a controlled trial in which oxygen equipment is allocated at random to a proportion of suitable subjects. This has not been carried out and would appear to be precluded by the immediate striking reduction in exertional dyspnoea which has been demonstrated under controlled conditions in subjects who benefit from oxygen. In any case, the effect on prognosis is likely to be small. Greater advantage may stem from deliberate reduction of activity (Donald, 1953; Harvey, Ferrer, and Cournand, 1953). However, patients to whom this might apply are already limited in what they can do, and for them the prospect of a short life but a merry one, while not offering much in the way of physicalactivity, may be a more attractive alternative.

\section{SUMMARY}

Two groups of 29 and 38 ex-coalworkers (with and without pneumoconiosis), disabled on account of chronic non-specific lung disease, have been investigated physiologically and followed for three and a half years and one year respectively. During these times $69 \%$ and $21 \%$ of the subjects died, the majority in respiratory failure. Measurements of indices of pulmonary function were made before follow-up at times when the subjects were as free from clinical bronchospasm, chest infection, and congestive cardiac failure as could be achieved by a period of hospital treatment and intensive therapy. The indices included arterial blood gas tensions at rest and on maximal exercise, the exercise response to oxygen breathing, and the single-breath pulmonary diffusing capacity.

The subjects with the poorest prognosis were those who initially had the greatest pulmonary ventilation perfusion inequality as judged $(\mathrm{P}<0.01)$ by a high percentage venous admixture, a low arterial blood oxygen tension at rest which fell further on maximal exercise, and the ability to perform more exercise breathing oxygen than air. Electrocardiographic evidence of pulmonary heart involvement was also a bad prognostic sign. Amongst subjects with an improvement in exercise performance on oxygen who also had right heart involvement, $50 \%$ died within 12 months. Some subjects were issued with portable oxygen equipment for use on exercise, but this did not influence prognosis.

Indices which had some prognostic value $(P<0.10)$ included the pulmonary diffusing capacity, the degree of hypercapnia on maximal exercise breathing oxygen, and the ventilation per unit of energy expenditure on exercise breathing air.

Indices of no prognostic value in this group of subjects included the maximum breathing capacity, the total lung volume, its subdivisions, and the residual volume as a percentage of total lung capacity, the blood erythrocyte sedimentation rate, and haemoglobin. The data lend limited support to the belief that a sheltered home life, reduced physical activity, and freedom from chest illness have a favourable influence upon prognosis.

This study has been made possible through the combined activities of many members of the P.R.U. working together as a team; to all of them I am grateful, especially Mrs. E. Venables, who got to know the patients in their homes, and Dr. R. S. Jones, who, with Mr. Meade, made the measurements of pulmonary diffusing capacity; Dr. D. Rivers, who made many of the post mortem examinations; Dr. T. G. Morris and Miss P Hewlett, who estimated blood lactic acid concentrations and the Misses J. I. Lovegrove, C. R. Scudder, and C. Yandle who contributed valuable technical assistance. I am also indebted to Dr. J. C. Gilson, Director of the Pneumoconiosis Research Unit, for his helpful interest, Mr. P. D. Oldham and Mr. C. Rossiter for statistical advice, and the subjects, many of whom collaborated in the belief that their contribution would be of value to others.

\section{REFERENCES}

Arnott, W. M. (1960). Lancet, 1, 1.

Baldwin, E. de F., Cournand, A., and Richards, D. W., Jr. (1949). Medicine (Baltimore), 28, 201.

Bates, D. V., Knott, J. M. S., and Christie, R. V. (1956). Quart. J. Med., n.s., 25, 137.

Berneus, B., Carlsten, A., Holmgren, A., and Seldinger, S. I. (1954). Scand. J. clin. Lab. Invest., 6, 217.

Cochrane, A. L., Davies, I., and Fletcher, C. M. (1951). Brit. J. industr. Med., 8, 244.

- Miall, W. E., Clarke, W. G., Jarman, T. F., Jonathan, G., and Moore, F. (1956). Brit. med. J., 1, 1193.

Cotes, J. E. (1960). In preparation.

- and Gilson, J. C. (1956a). Lancet, 1, 872. (1956b). Ibid., 2, 823.

and Meade, F. (1960). Ergonomics, 3, 97.

-- and Oldham, P. D. (1959). J. appl. Physiol., 14, 467.

Cournand, A. (1957). Science, 125, 1231.

Donald, K. W. (1953). Brit. med. J., 1, 415.

Fletcher, C. M. (1952). Proc. roy. Soc. Med., 45, 577.

- (1959). Thorax, 14, 286.

Forster, R. E., Cohn, J. E., Briscoe, W. A., Blakemore, W. S., and Riley, R. L. (1955). J. clin. Invest., 34, 1417.

- Fowler, W. S., Bates, D. V., and Van Lingen, B. (1954). Ibid. 33, 1135 . 
Gandevia, B., and Hugh-Jones, P. (1957). Thorax, 12, 290. Gilson, J. C., and Hugh-Jones, P. (1949). Clin. Sci., 7, 185. (1955). Spec. Rep. Ser. med. Res. Coun. (Lond.) 290.

Gough, J., and Wentworth, J. E. (1949). J. roy. micr. Soc., 69, 231. Halmagyi, D. F., and Cotes, J. E. (1959). Clin. Sci., 18, 475. Harvey, R. M., Ferrer, M. I., and Cournand, A. (1953). Circulation, 7, 932 .

Jones, R. S., and Meade, F. (1960). Lancet, 1, 94.

Kennedy, M. C. S. (1953). Thorax, 8, 73.

King, E. J. (1947). Biochem. J., 41, xxxii.

Leopold, J. G., and Gough, J. (1957). Thorax, 12, 219.

McKerrow, C. B., McDermott, M., and Gilson, J. C. (1960). Lancet, $1,149$.

Ogilvie, C. (1959). Thorax, 14, 113.

Platts, M. M., and Greaves, M. S. (1957). Ibid., 12, 236.
Riley, R. L., Campbell, E. J. M., and Shepard, R. H. (1957). J. appl. Physiol., 11, 245.

and Cournand, A. (1951). Ibid., 4, 77.

Permutt, S., Said, S., Godfrey, M., Cheng, T. O., Howell, J. B. L., and Shepard, R. H. (1959). Ibid., 14, 339.

Roughton, F. J. W., and Forster, R. E. (1957). Ibid., 11, 290.

Scholander, P. F. (1947). J. biol. Chem., 167, 235.

Shepard, R. H., Cohn, J. E., Cohen, G., Armstrong, B. W., Carroll, D1. G., Donoso, H., and Riley, R. L.' (1955). Amer. Rev. Tuberc.,

Tubercle (Lond.), 39, 307.

Sinclair, J. D. (1955). Brit. J. Tuberc., 49, 157.

Tenney, S. M., and Miller, R. M. (1956). J. appl. Physiol., 9, 321.

Thomas, A. J. (1951). Brit. Heart J., 13, 1. (1955). Med. Press, 234, 437.

West, J. R., Baldwin, E. de F., Cournand, A., and Richards, D. W. (1951). Amer. J. Med., 10, 481. 